\title{
The Evaluation Acute Traumatic Stress Level in Close Relatives of Stroke Patients
}

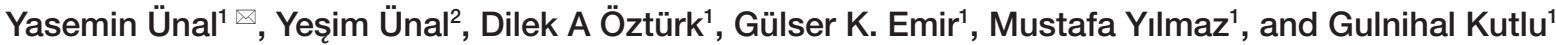 \\ ${ }^{1}$ Department of Neurology, Muğla Sıtkı Koçman University Faculty of Medicine, Muğla, Turkey \\ ${ }^{2}$ Ministry of Health Istanbul Provincial Health Directorate, Istanbul, Turkey
}

Objective Stroke is the second most common cause of death and the leading cause of adult disability. Both stroke patients and their family can therefore experience increased traumatic stress level.

Methods The participants are close relatives of patients $(n=65)$ who had a first time stroke (CRPWS) hospitalized. A control group (CG) ( $n=61$ ), who had no history of chronic illness in their family and had at least one traumatic life event experience. The National Institutes of Health Stroke Scale, Modified Rankin Scale, Personal Information Form, Life Events Checklist, Traumatic Stress Symptom Scale, and Multidimensional Scale of Perceived Social Support, were used in the study.

Results We found no significant association between NIHSS and MRS of patients and traumatic stress level of the family member. The traumatic stress level was significantly higher in the CRPWS group than in the CG group. Traumatic stress level was higher in women than men and was not associated with perceived social support in the CRPWS group.

Conclusion The traumatic stress level of the relatives was not associated with the clinical features of the stroke patients. In the early phase, after the diagnosis of stroke, psychological support may be important to prevent CRPWS from PTSD.

Psychiatry Investig 2017;14(5):546-549

Key Words Acute posttraumatic stress disorder, Secondary traumatic stress.

\section{INTRODUCTION}

Stroke is the second most common cause of death and the leading cause of disability in adults. ${ }^{1}$ Unpredictable events such as stroke may psychologically affect both close relatives and people who witnessed the event of stroke. Traumatic stress level may increase after the stroke event as a psychological consequence of stroke for both the patients with stroke (PWS) and their close family members. Traumatic stress level or the post traumatic stress disorder for caregivers of the PWS has been examined in many studies. ${ }^{2-4}$ However, the acute stress level of family members of PWS has not been evaluated thoughtfully. In our study, we investigated the acute stress level of close relatives of PWS in the acute phase of stroke and

Received: July 1, 2016 Accepted: August 19, 2016

Available online: May 16, 2017

$\triangle$ Correspondence: Yasemin Ünal, MD

Department of Neurology, Mugla Sitki Kocman University, Faculty of Medicine, Orhaniye Neighborhood Haluk Özsoy street, 48000 Mugla, Turkey

Tel: +905052553435, Fax: +902522111345

E-mail: yaseminunal95@yahoo.com

(a) This is an Open Access article distributed under the terms of the Creative Commons Attribution Non-Commercial License (http://creativecommons.org/licenses/bync/4.0) which permits unrestricted non-commercial use, distribution, and reproduction in any medium, provided the original work is properly cited. we also analyzed factors which may affect it.

The reactions of family member of PWS, a life-threatening illness, may consistent with traumatic stress responses. ${ }^{5}$ Traumatic stress symptoms consist of intrusive thoughts, emotional numbing, physiological arousal and avoidance. ${ }^{5}$ For the diagnosis of Acute Stress Disorder (ASD), specific symptoms must occur within 2-28 days following the traumatic event. Subclinical symptoms of acute stress are also possible during this time period. ${ }^{5,6}$ When the symptoms last more than 28 days, it may be refer red to as post traumatic stress disorder (PTSD). ${ }^{6}$

The aims of our study were as following:

- To examine the acute stress level of family members of newly diagnosed stroke patients.

- To determine predictors of traumatic stress symptoms and the correlation between demographic variables and traumatic stress symptoms.

To identify traumatic stress early may be useful for formulating psychosocial support for the PWS and increase their quality of life as a result of long-term well being of the caregivers. 


\section{METHODS}

Between January and July 2015 a total of 126 subjects were included in the study. Close relatives of patients who had their first episode stroke (CRPWS), and were hospitalized in Muğla Sttkı Koçman University Research and Training Hospital, Turkey. We divided CRPWS in two group: close relatives that take caregiving or not. A control group (CG) (n= 61), who had no chronic illness history in their family and had at least one traumatic life event experience were also included in the study. This study was designed according to the principles of the Helsinki Declaration and approved by the local ethical committee. All participants agreed and signed the informed consent prepared by the researchers.

The stroke was diagnosed after a neurological examination and CT and/or MRI examination. The National Institutes of Health Stroke Scale (NIHSS) and the Modified Rankin Scale (MRS) were used to determine the clinical severity of PWS.

All participants completed a questionnaire with information about age, gender, socio-economic status, marital status, educational level, pre-mental illness story and closeness as a caregiver relative to PWS. Participants, who had a diagnosed depression or an anxiety disorder history, were excluded from the study.

To determine the traumatic events experienced by all participants we used the Turkish version of Life Event Checklist (LEC), a part of the Clinician Administered Post Traumatic Stress Disorder Scale (CAPS). ${ }^{7}$ Aker et al. ${ }^{8}$ made the validity and reliability of the test to our culture. The Life Event Checklist (LEC) consists of 17 items, and 16 of the items are known to cause PTSD symptoms and the last item require exposure to other events.

The Traumatic Stress Symptom Scale (TSSS) was used to determine the traumatic stress level. ${ }^{9}$ The scale consists of 23 items, 17 items are associated with post-traumatic stress disorder and 6 items for identifying symptoms of depression. The Turkish version of the Multidimensional Scale of Perceived Social Support (MSPSS) was used in order to determine the level of social support. ${ }^{10,11}$

The relatives answered the questionnaires during study weeks after the stroke occurred. They were instructed to answer the questions as if they just learned or witnessed the of stroke diagnosis. The participants in the control group answered the questionnaire according to the traumatic events experienced earlier in life. Statistical analysis was performed using the Statistical Package for the Social Sciences program (SPSS 21.0 for Windows, IBM Corp., Armonk, NY, USA). Descriptive analysis (mean, frequency), independent sample t-test and the Pearson product-moment correlation were used for the analysis. A p-value less than 0.05 were considered significant.

\section{RESULTS}

The average age for PWS $(n=54)$ was $70 \pm 12$ years of age. Thirty-two PWS were female and 22 patients were male. Forty-nine of the patients were diagnosed with an ischemic and five had a hemorrhagic stroke. The average score of the National Institutes of Health Stroke Scale of stroke patients were $7.1 \pm 6.3$ and the Modified Rankin Scale were 3.4 \pm 1.4 .

The mean age for the CRPWS were $50( \pm 10)$ years of age and for the control group the men age was $40( \pm 10)$ years of age. There were 31 females and 34 men in the group of CRPWS and the control group 39 were female and 22 were male. The demographic data for the CRPWS group and for the control group are presented in the Table 1.

We found a significant higher traumatic stress level for the CRPWS group compared with the control group $(\mathrm{p}<0.001)$. However, there was no significant difference between the two

Table 1. The demographic data of CRPWS and CG

\begin{tabular}{|c|c|c|}
\hline $\begin{array}{c}\text { Demographic properties } \\
\text { of the participants }\end{array}$ & CRPWS, N (\%) & CG, N (\%) \\
\hline \multicolumn{3}{|l|}{ Gender } \\
\hline Female & $31(47.7)$ & $39(36.1)$ \\
\hline Male & $34(52.3)$ & $22(63.9)$ \\
\hline \multicolumn{3}{|l|}{ Marital status } \\
\hline Single & $2(3.1)$ & $12(19.7)$ \\
\hline Married & $51(80.0)$ & $49(80.3)$ \\
\hline Widow & $11(16.9)$ & 0 \\
\hline \multicolumn{3}{|l|}{ Educational status } \\
\hline No literate & $4(6.3)$ & $1(1.6)$ \\
\hline Literate & $6(9.4)$ & 0 \\
\hline Primary education & $27(42.2)$ & $2(3.3)$ \\
\hline High school & $12(18.8)$ & $12(19.7)$ \\
\hline University & $13(20.3)$ & $36(59.0)$ \\
\hline Graduate & $2(3.1)$ & $8(13.1)$ \\
\hline Other & 0 & $2(3.3)$ \\
\hline \multicolumn{3}{|l|}{ Mental disease } \\
\hline Yes & $8(12.7)$ & $5(8.2)$ \\
\hline No & $55(87.3)$ & $56(91.8)$ \\
\hline \multicolumn{3}{|l|}{ History of addiction } \\
\hline No & $35(64.8)$ & $48(78.7)$ \\
\hline Cigarette & $17(31.5)$ & $13(21.3)$ \\
\hline Alcohol & $1(1.9)$ & 0 \\
\hline Other & $1(1.9)$ & 0 \\
\hline \multicolumn{3}{|c|}{ History of mental disease in family } \\
\hline Yes & $8(13.3)$ & $4(6.6)$ \\
\hline No & $52(86.7)$ & 57 (93.4) \\
\hline
\end{tabular}


Table 2. Traumatic Stress and Social Support Level of CRPWS and $C G$

\begin{tabular}{lrrrrr}
\hline $\begin{array}{l}\text { Traumatic Stress } \\
\text { and Social } \\
\begin{array}{c}\text { Support Level } \\
\text { of participants }\end{array}\end{array}$ & Mean & SD & & Mean & SD \\
\cline { 2 - 3 } \cline { 5 - 6 } \cline { 5 - 6 } TSSS & 19.984 & 14.218 & & 11.409 & 10.638 \\
MSPSS & 63.769 & 21.429 & & 67.901 & 16.499 \\
Family & 22.523 & 7.560 & & 23.409 & 5.559 \\
Friend & 20.907 & 7.307 & & 21.934 & 6.185 \\
Private person & 20.338 & 8.943 & 22.557 & 7.292 \\
\hline
\end{tabular}

CRPWS: close relatives of patients with stroke, CG: control group, TSSS: The Traumatic Stress Symptom Scale, MSPSS: Multidimensional Scale of Perceived Social Support

groups in terms of the level of social support perceived from relatives or other persons. The results are presented in Table 2.

The traumatic stress level of the female CRPWS was significantly higher than for the male CRPWS $(\mathrm{p}=0.029)$. We found no association between the traumatic stress level and the perceived social support in the CRPWS group. The level of perceived social support from family was higher for the male CRPWS ( $\mathrm{p}=0.022$ ) than for the female CRPWS. The level of traumatic stress was significantly reduced when their perceived social support level from family were increased in the CRPWS group ( $\mathrm{p}=0.038$ ). There was no significant difference with the traumatic stress level and the social support level between females and males in the control group.

The traumatic stress level was higher in females of the CRPWS group ( $\mathrm{p}<0.001$ ). The social support level was lower, both total social support $(\mathrm{p}=0.02)$, social support from family $(\mathrm{p}=$ 0.02 ) and social support from private persons $(\mathrm{p}=0.02)$, in females of the CRPWS group than for the females in the CG. There was no significant difference between males in the CRPWS group and males in the CG on the traumatic stress level and the perceived social support.

We found no significant correlation between NIHSS and MRS scores of patients and traumatic stress level of the CRPWS. When the participants in the CRPWS group were divided in to caregivers or not, the traumatic stress level and the total social support levels were not significantly different between the two groups.

\section{DISCUSSION}

We found not surprisingly, that the acute traumatic stress level was significantly higher in the CRPWS than in the CG. Stroke has a high mortality and morbidity and with a sudden onset. Early in the cerebrovascular disease, the patient's physical and cognitive status may prevent direct communication with the health professionals. ${ }^{12}$ The close family members are most probably the first communicating with the health professionals, for information and sometimes for critical treatment decisions. A good contact between relatives and health professionals are important for the treatment and care of the patient, and provides psychological and physical support to the patient. ${ }^{12}$ If the relatives stress and anxiety level are high, it may affect an effective communication, which may cause problem in the treatment of the patient. ${ }^{12,13}$ To be aware of high stress level of the family members may help the physicians to a better and clear communication with the caregivers. ${ }^{12}$

Stroke as one of the major causes of disability gives both the patient and caregivers significant adverse effects. ${ }^{2,4}$

Previous studies showed that high emotional distress of the caregivers are associated with lower functional status of the patient. $^{2}$

We found no significant correlation between the traumatic stress level of the CRPWS and NIHSS, MRS scores and the clinical severity of the patients. It could be explained that, the close relatives may not realize these verities of stroke in the early phase just after diagnosis of stroke. Similarly, it's found that the child's medical condition is not associated with severity of psychological reactions of their parents in some studies. ${ }^{14}$

There was no significant difference between the level of traumatic stress and perceived social support between the subgroups of CRPWS. Being a close relative of a stroke patient may on itself be connected with acute stress reactions, independently of the burden of care by the relatives. The relatives may not realize and may foreseen the difficulties of care burden after hospitalization in the acute phase of the disease.

Traumatic stress level was higher in female CRPWS than the male CRPWS and female in CG. A high stress level in women has been found in earlier studies. ${ }^{12,15}$

Social support has been reported as an important factor in coping with stress and has a protective role of the negative effects of anxiety..$^{16-18}$ An inverse relationship between perceived social support and traumatic stress is highlighted in previous studies in the literature. We found no difference in terms of social support between CRPWS and control group. There was also no difference between the CRPWS and the control group in terms of female gender. This finding showed that the effect of social support in terms of acute traumatic stress was insufficient or insignificant. In the acute phase, just after the traumatic event, it may also be important to assess other factors than social support. The perceived social support, family score, was significantly higher in males than females in the CGPWS group. An explanation of this finding may be that most of the caregivers of the stroke patients in our culture are females. On the other hand, in an earlier study it was highlighted that perceiving social support was a subjective issue rather than 
being objective. ${ }^{19}$ In the assessment, perception of social support has not occurred enough in early stages of the disease.

The relationship between traumatic stress and perceived social support has not been varied according to the level of education and income status. After traumatic events, the posttraumatic stress level appears to be higher in persons who are single, widowed or living alone, because their social support is less or not enough. ${ }^{20}$ In our study, there was no difference between married or single subjects regarding traumatic stress levels. However, social support level was higher with married people.

We found no significant difference if the health professionals, relatives or other persons had knowledge about the disease or not. However, we did not investigate if the relatives were in an interactive communication with the health professionals or not. We also did not study the importance of correct transfer of information about the clinical situation of the patients. This is an issue for future studies.

ASD is a common sequele following traumatic events, and a significant proportion can develop PTSD. ${ }^{21}$ In the early phase, after the traumatic event, psychological support by relatives is a key factor to prevent the patient from developing PTSD. Several supportive strategies, such as brief cognitive behavioral therapy, supportive counseling, exposure based therapy or pharmacological therapy, could be useful for preventing the development of PTSD. ${ }^{22,23}$

We did not examine which acute stress symptoms that gave PTSD or the effect of supportive counseling to prevent PTSD in a long-term process.

Health professionals should be informed that they play a major role in preventing PTSD to develop in the CRPWS and they should be trained to detect stress reactions in the CRPWS.

In future studies, it can be investigated how many of the CRPWS with high acute stress levels will develop post-traumatic stress disorder and what the prognosis. Many family members are suffering following the stroke diagnosis and psychosocial support at diagnosis remains critically important.

The study has some limitations. Self-reported traumatic stress scale was used instead of clinician administrated ASD evaluating form. There is no cultural adapted clinician administrated ASD scale. It's highly recommended to develop or adapt clinician administrated ASD form in to the Turkish language.

Most significantly, the data are cross-sectional in nature and it had been better to re-assess the participants after one or more month, to evaluate the traumatic stress responses of the patients.

\section{REFERENCES}

1. World Health Organization. Available at: http://www.who.int/media- centre/factsheets/fs310/en/. Accessed April, 2016.

2. Jaracz K, Grabowska-Fudala B, Kozubski W. Caregiver burden after stroke: towards a structural model. Neurol Neurochir Pol 2012;46:224232.

3. Holmes AM, Deb P. The effect of chronic illness on the psychological health of family members. J Ment Health Policy Econ 2003;6:13-22.

4. Simith-Johnson B, Davis BL, Burns D, Montgomery AJ, McGee ZT. African American Wives and perceived stressful experiences: providing care for stroke survivor spouses. ABNF J 2015;26:39-42.

5. Patino-Fernandez AM, Pai AL, Alderfer M, Hwang WT, Reilly A, Kazak AE. Acute stress in parents of children newly diagnosed with cancer. Pediatr Blood Cancer 2008;50:289-292.

6. American Psychiatric Association. Diagnostic and Statistical Manual of Mental Disorders, Fifth Edition. Washington, DC: American Psychiatric Association; 2013.

7. Blake DD, Weathers FW, Nagy LM, Kaloupek DG, Gusman FD, Charney DS, et al. The development of a clinician-administered PTSD Scale. J Trauma Stress 1995;8:75-90.

8. Aker AT, Özeren M, Başoğlu M, Kaptanoğlu C, Erol A, Buran B. Clinician Administered Post Traumatic Stress Disorder Scale (CAPS) reliability and validity study. Turk J Psychiatry 1999;10:286-293.

9. Başoğlu M, Şalcıŏglu E, Livanou M, Özeren M, Aker T, Kilıç C, et al. A study of the validity of a screening instrument for traumatic stress in earthquake survivors in Turkey. J Trauma Stress 2001;14:491-509.

10. Zimet GD, Powell SS, Farley GK, Werkman S, Berkoff KA. Psychometric characteristics of the multidimensional scale of perceived social support. J Pers Asses 1990;55:610-617.

11. Eker D, Arkar H, Yaldız H. Factorial structure, validity, and reability of Revised form of the multidimensional scale of perceived social support. Turk J Psychiatry 2001;12:17-25.

12. Scrimin S, Haynes M, Altoe G, Bornstein MH, Axia G. Anxiety and stress in mothers and fathers in the $24 \mathrm{~h}$ after their child's surgery. Child Care Health Dev 2009;35:227-233.

13. Montgomery C, Lydon A, Lloyd K. Psychological distress among cancer patients and informed consent. J Psychosom Res 1999;46:241-245.

14. Muscara F, McCarthy MC, Woolf C, Hearps SJ, Burke K, Anderson VA. Early psychological reactions in parents of children with a life threatening illness within a pediatric hospital setting. Eur Psychiatry 2015;30:555-561.

15. Favrole P, Jehel L, Levy P, Descombes S, Muresan IP, Manifacier MJ, et al. Frequency and predictors of post-traumatic stress disorder after stroke: a pilot study. J Neurol Sci 2013;327:35-40.

16. Dunn ME, Burbine T, Bowers CA, Tantleff-Dunn S. Moderators of stress in parents of children with autism. Community Ment Health J 2001; 37:39-52.

17. Berterö CM. Types and sources of social support for people afflicted with cancer. Nurs Health Sci 2000;2:93-101.

18. Weiss MJ. Harrdiness and social support as predictors of stress in mothers of typical children, children with autism, and children with mental retardation. Autism 2002;6:115-130.

19. Tural Ü, Coşkun B, Önder E, Çorapçıŏlu A, Ylldız M, Kesepara C, et al. Psychological Consequences of the 1999 Earthquake in Turkey. J Trauma Stress 2004;17:451-459.

20. Silva JA, Derecho DV, Leong GB, Weinstock R, Ferrari MM. A classification of psychological factors leading to violent behavior in posttraumatic stress disorder. J Forensic Sci 2001;46:309-316.

21. Suliman S, Troeman Z, Stein DJ, Seedat S. Are neuropsychological deficits after trauma associated with ASD severity? Compr Psychiatry 2014; 55:145-154.

22. Forneris CA, Gartlehner G, Brownley KA, Gaynes BN, Sonis J, CokerSchwimmer E, et al. Interventions to prevent post-traumatic stress disorder: a systematic review. Am J Prev Med 2013;44:635-650.

23. Nixon RD. Cognitive processing therapy versus supportive counseling for acute stress disorder following assault: a randomized pilot trial. Behav Ther 2012;43:825-836. 